\title{
Integrating Body Scanning Solutions into Virtual Dressing Rooms
}

\author{
Francesco Sapio \\ Sapienza University of Rome, Italy \\ Rome, Italy \\ sapio@diag.uniroma1.it
}

\author{
Andrea Marrella \\ Sapienza University of Rome, Italy \\ Rome, Italy \\ marrella@diag.uniroma1.it
}

\author{
Tiziana Catarci \\ Sapienza University of Rome, Italy \\ Rome, Italy \\ catarci@diag.uniroma1.it
}

\begin{abstract}
The world is entering its 4th Industrial Revolution, a new era of manufacturing characterized by ubiquitous digitization and computing. One industry to benefit and grow from this revolution is the fashion industry, in which Europe (and Italy in particular) has long maintained a global lead. To evolve with the changes in technology, we developed the IT-SHIRT project. In the context of this project, a key challenge relies on developing a virtual dressing room in which the final users (customers) can virtually try different clothes on their bodies. In this paper, we tackle the aforementioned issue by providing a critical analysis of the existing body scanning solutions, identifying their strengths and weaknesses towards their integration within the pipeline of virtual dressing rooms.
\end{abstract}

\section{CCS CONCEPTS}

- Human-centered computing $\rightarrow$ Virtual reality; Gestural input;

\section{KEYWORDS}

Virtual Dressing Room, Body Scanning, IT-SHIRT

\section{ACM Reference Format:}

Francesco Sapio, Andrea Marrella, and Tiziana Catarci. 2018. Integrating Body Scanning Solutions into Virtual Dressing Rooms. In Preprint of 2018 International Conference on Advanced Visual Interfaces, AVI '18, May 29-fune 1, 2018, Castiglione della Pescaia, Italy. ACM, New York, NY, USA, 3 pages. https://doi.org/10.1145/3206505.3206589

\section{INTRODUCTION}

In the context of digital dressing rooms, the Italian project IT-SHIRT (IT-empowering faSHIon design creators with a next generation cocReation ecosysTem) aims at providing customers with the ability to experiment with alternative fashion products in real time as their body type is detected. The project is driven by the consideration that the European fashion industry needs to make digital technologies an integral part of the fashion value chain in order to retain its global lead. The final objective of the project is to introduce tangible ways to make such integration possible.

Compared to traditional approaches that are entirely based on physical models, the "digital aspect" becomes an accelerating factor that facilitates collaboration between designers, minimizes the

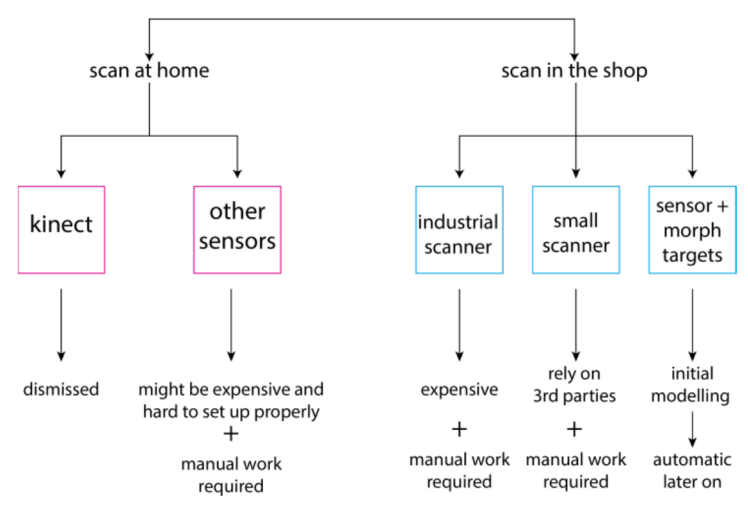

Figure 1: Different approaches to virtual dressing rooms.

time-to-market, and enables immediate consumer feedback. Current state-of-the-art technologies in 3D-reconstruction, clothing simulation and substituted reality can be combined to optimize the realism of the experience of interacting and experimenting with virtually prototyped materials and clothes. For example, the unified framework presented by Macklin et al. [6] employs Position-Based Dynamics (PBD) techniques as a building block to model in real time the animation of gases, liquids, deformable solids and rigid bodies, including interactions and collisions with one another. In addition, further well-known solutions are available, among the others, in the open source Bullet physics engine [2] and in the proprietary PhysX SDK [5], which are both employed in the development of video games for the most popular gaming platforms.

In the context of IT-SHIRT, we aim at investigating the development of tools for user body measurements starting from body scanning techniques. Such tools will be integrated with online and offline fashion digital dressing rooms, which will allow to showcase the digital designs and collect feedbacks from consumers. In the long term, we will assess the practical utility of the solutions analyzed during the project against the challenging Italian fashion industry and in international fashion initiatives. In this paper, we start identifying and discussing different solutions to integrate body scanning within the virtual dressing room pipeline.

\section{BODY SCANNING SOLUTIONS}

The body scanning task is key in a virtual dressing room in which the final users (customers) can see themselves trying clothes on with their own bodies. Placing this step rightly within the pipeline adds an intrinsic value to virtual dressing, rather than an extra overhead. In particular, as shown in Figure 1, we have been focusing on two different kind of approaches. The first approach consists 


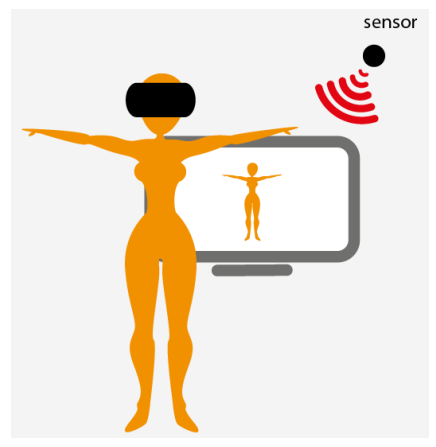

Figure 2: Conceptualization of scanning at home.

of allowing the customer to do body measurements at home (see Figure 2), before entering the virtual dressing room. The advantages of this solution are that the user is in control of her/his own body shape. In case the user changes her/his body, the system can easily re-measure it. In addition, the system can keep track of the user's body over time by providing insightful statistics. In the second approach, the customer goes into a physical shop, in which a body scanning technology takes the measurements (controlled by a human operator), and uploads those to a server (see Figure 3). In a second moment, the customer can log in at home in the virtual dressing room and download the digital counterpart of her/his body shape, which can be used to try out clothes.

\section{SCANNING AT HOME}

Scanning at home requires users to employ dedicated hardware with which they can take the measurements of their bodies. The Kinect sensor [9] by Microsoft would be a suitable candidate to perform the scanning task at home. However, since it has been recently dismissed, its selection can be considered as an unwise choice for the objectives of our research, despite it is acknowledged that it can be potentially useful to tackle several challenges in body scanning (e.g., $[1,3,4,8])$. Other solutions require the users to employ RGBD (Red Green Blue Depth) camera sensors, such as the SteroLab's camera ZED (cf. https://www.stereolabs.com/zed/) or the Orbbec's camera Astra (cf. https://orbbec3d.com/). They both provide a Software Development Kit (SDK), but neither of them is specifically tailored for body scanning. Finally, we notice that to achieve a level of precision that is suitable for virtual dressing rooms, a valid solution should provide Machine Learning techniques relying on the construction of a large initial dataset of bodies. However, to date, none of the above solutions enables to integrate such techniques.

\section{SCANNING IN SHOPS}

Scanning in shops allows some extra flexibility in the choice of suitable hardware to perform the scanning task. An interesting solution is to use industrial size scanners, such as Artect3D's Eva, SpiderSpace and Leo (cf. https://www.artec3d.com/) or Creaform's GoScan! (cf. https://www.creaform3d.com/). In spite of their high cost and the need of a special day-training for learning their usage, such solutions are born with the purpose of scanning objects and not human beings. As a result, the time and effort required to perform

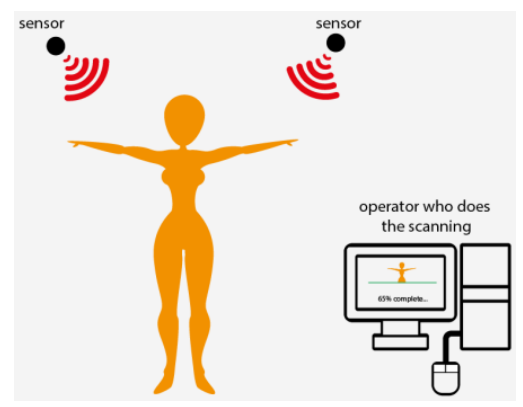

Figure 3: Conceptualization of scanning in shops.

the scanning task, coupled with additional efforts for manually adjusting the models after the scanning, made the industrial sized scanners not a suitable candidate for our research.

Other solutions, namely ShapeScale (cf. https://shapescale.com) and StructuredSensor (cf. https://structure.io/), were developed in the form of small and portable systems possibly tailored for body scanning. The usability and the reduced cost, compared with suitable results for virtual dressing rooms, make such systems as one of the best solutions for performing scanning in shops. However, one downside is that not all of them have an available open SDK for development, making very tricky their practical usage.

A final solution consists of building a custom sensor network, which could reconstruct the shape of an individual's body. In particular, we have been investigating a network of sensors to capture some core measures of the human body, and plug them into specific predefined 3D models with Morph Targets (a method used to deform a 3D mesh). As a result, by adjusting the values of the Morph Targets, it is possible to reconstruct a body shape with enough precision for a virtual dressing room. Moreover, the use of high-end virtual reality solutions (e.g., Oculus Rift along with Oculus Touch or VIVE Headset along with its controllers) allows to add extra parameters, such as height and arm length, which can be used to calibrate and be fed into Morph Targets as well. Note that although recreating a model involves an initial modelling effort (with all the consequent issues, some of them explained in [7]), it will allow to completely automate the modeling process over time, reducing any manual effort for the elaboration of the models.

\section{DISCUSSION}

Our analysis of existing scanning solutions to be integrated with virtual dressing rooms has led us to: (i) identify the use of a small network of sensors, coupled with dedicate software, as the most natural choice to build a sufficient precise digital body shape starting from the measured data; and (ii) understand that the approach in which the user performs the scanning task at home does not fit well with the project purposes due to the many limitations implied. Conversely, allowing customers to build their digital body shape in a physical shop and then download it at home at a later time seems to be the right trade-off to make feasible the concrete use of virtual dressing rooms in fashion industry.

Acknowledgments. This work is partly supported by the Italian project IT-SHIRT (Sapienza grant). 
Integrating Body Scanning Solutions into

Virtual Dressing Rooms

\section{REFERENCES}

[1] Ke-Li Cheng, Ruo-Feng Tong, Min Tang, Jing-Ye Oian, and Michel Sarkis. 2016. Parametric human body reconstruction based on sparse key points. IEEE transactions on visualization and computer graphics 22, 11 (2016), 2467-2479.

[2] Erwin Coumans. 2015. Bullet physics simulation. In ACM SIGGRAPH 2015 Courses. ACM, 7.

[3] Nils Hasler, Carsten Stoll, Bodo Rosenhahn, Thorsten Thormählen, and HansPeter Seidel. 2009. Estimating body shape of dressed humans. Computers \& Graphics 33, 3 (2009), 211-216.

[4] Furkan Isikdogan and Gokcehan Kara. 2012. A real time virtual dressing room application using Kinect. CMPE537 Computer Vision Course Project (2012).
AVI '18, May 29-June 1, 2018, Castiglione della Pescaia, Italy

[5] WEI Li-xin. 2009. On the Physics Engine to Use the PhysX SDK. Computer Knowledge and Technology 5, 20 (2009), 5561-5562.

[6] Miles Macklin, Matthias Müller, Nuttapong Chentanez, and Tae-Yong Kim. 2014. Unified particle physics for real-time applications. ACM Transactions on Graphics (TOG) 33, 4 (2014)

[7] Nadia Magnenat-Thalmann, Frederic Cordier, Hyewon Seo, and George Papagianakis. 2004. Modeling of bodies and clothes for virtual environments. In 2004 International Conference on Cyberworlds. IEEE, 201-208.

[8] Jing Tong, Jin Zhou, Ligang Liu, Zhigeng Pan, and Hao Yan. 2012. Scanning 3d full human bodies using kinects. IEEE transactions on visualization and computer graphics 18, 4 (2012), 643-650.

[9] Zhengyou Zhang. 2012. Microsoft kinect sensor and its effect. IEEE multimedia 19, 2 (2012), 4-10. 